\title{
Genetic diversity of Ralstonia solanacearum phylotype II sequevar 4 strains associated with Moko disease of banana (Musa spp.) in Peninsular Malaysia
}

\begin{abstract}
Moko disease caused by Ralstonia solanacearum (R. solanacearum) is a major disease affecting banana (Musa spp.) production. Although local reports suggested that this disease is widespread in Malaysia, molecular characterization of R. solanacearum strains associated with Moko disease in this country has not yet been done. During March 2011 to June 2012, 170 banana plants associated with Moko disease and the adjacent soil samples were collected in 12 different locations of five outbreak states in Peninsular Malaysia comprising Kedah, Selangor, Pahang, Negeri Sembilan and Johor, with disease incidence exceeding $80 \%$ in some severely affected plantations. A total of 197 strains were identified as R. solanacearumlike colonies since they produced fluidal colonies that were white to pink coloration after incubation at 24 to $48 \mathrm{~h}$ at $29^{\circ} \mathrm{C}$ on Kelmanôs TZC agar medium, appeared as Gram-negative rods, and positive for potassium hydroxide $(\mathrm{KOH})$, Kovacs oxidase, catalase and lipase activity on Tween 80 solution tests. Biovar tests disclosed that only 30 strains displayed characteristics of biovar $1 \mathrm{R}$. solanacearum associated with Moko disease, which was negative for utilization of disaccharides and hexose alcohols. Pathogenicity assay showed that these 30 strains were virulence towards Musa paradisiaca cv. Nipah explants with diverse degrees of virulence. Phylotype-specific multiplex PCR (Pmx-PCR) revealed all strains belonged to phylotype II displaying a 372 bp amplicon. Phylogenetic analyses of endoglucanase (egl) sequences clustered all 30 strains into phylotype II/4, together with the reference sequences strains from Peru (UW129, UW162 and UW163) and Colombia (UW070). Pooled rep-PCR fingerprinting method defined two major groups; cluster 1 (subgroup A and B) and cluster 2 (sub-group C), with $35 \%$ average similarity coefficient within these two clusters. The sub-groups in cluster 1 were represented by strains from Kedah, Selangor, Negeri Sembilan and Johor; while cluster 2 sub-group was represented exclusively by strains of Pahang. To our knowledge, this is the first description of R. solanacearum phylotype II/4 in Malaysia and the Asian region. Our findings may expand constructive documentation and describe a better understanding on diversity of Malaysian $R$. solanacearum Moko-causing strains populations, thus will be useful for designing disease control strategies.
\end{abstract}

Keyword: Banana; Genetic diversity; Moko disease; Phylotyping; Ralstonia solanacearum strains; Rep-PCR; Sequevar 